\title{
Standardized versus research-based PAM50 intrinsic subtyping of breast cancer
}

\author{
A. Prat ${ }^{1,2,3}$ - J. S. Parker ${ }^{4,5}$ \\ Received: 7 July 2019 / Accepted: 12 August 2019 / Published online: 21 August 2019 \\ ๑) Federación de Sociedades Españolas de Oncología (FESEO) 2019
}

To date, there is only one standardized assay for identification of the so-called intrinsic molecular subtypes of breast cancer (i.e., Luminal A, Luminal B, HER2-enriched, and Basal-like) described by Perou et al. [1]. This assay uses the nCounter technology, has been CE Marked, and is commercially available worldwide [2]. Over the years, however, several research-based methods for subtype identification have been reported [3-5]. However, none of them is standardized and, in most cases, proper controls or data to suggest that the method is accurate and precise compared to the standardized and clinically validated nCounter-based PAM50 assay are missing [6, 7]. This is worrisome, since important conclusions regarding the prognostic or predictive value of the PAM50 subtypes are being drawn from "homebrew" research-based PAM50 versions often run on different technologies.

In 2009, Parker and colleagues proposed the use of a standardized 50-gene set (PAM50) for subtype classification to improve the classification concordance reported by investigators [3]. However, a gene set does not completely resolve discrepancies between researchers, since the genes might be quantitatively measured using different algorithms, technologies, and/or normalization methods $[8,9]$. It is important to remember that the PAM50 assay is based on a single sample predictor (SSP), meaning that the algorithm and any parameter values are exclusively determined from a

\author{
A. Prat \\ alprat@clinic.cat \\ 1 Department of Medical Oncology, Hospital Clinic de \\ Barcelona, Barcelona, Spain \\ 2 Translational Genomics and Targeted Therapeutics in Solid \\ Tumors, IDIBAPS, Barcelona, Spain \\ 3 SOLTI, Barcelona, Spain \\ 4 Lineberger Comprehensive Cancer Center, University \\ of North Carolina at Chapel Hill, Chapel Hill, NC, USA \\ 5 Department of Genetics, University of North Carolina \\ at Chapel Hill, Chapel Hill, NC, USA
}

training set, and test cases are assessed independently. This requires that measurements and normalizations of a test case are not dependent on other test cases; in reality, this means that the same methods are used in both the training and testing predictions (as is done in the nCounter-based PAM50, Oncotype DX [Genomic Health, USA] and Agendia Mammaprint assays [Agendia, The Netherlands]). This is because using different technologies (i.e., nCounter vs RNAseq vs qRT-PCR) exhibit measurement bias. In addition, different analytic methods used within the same platform will also exhibit measurement bias. The only way to reduce measurement bias, whether you are using the same platform or another one, is using controls; however, controls are normally unavailable.

One particular contemporary example is the recent report by Turner et al. [10]. In their study, they performed gene expression profiling using RNA-sequencing from 302 (58\%) tumor samples of the PALOMA-3 phase III clinical trial [11], which randomized patients with advanced or metastatic aromatase inhibitor (AI)-resistant hormone receptor-positive $(\mathrm{HR}+) / \mathrm{HER} 2-$ negative breast cancer to fulvestrant + palbociclib or fulvestrant + placebo. The main finding was that high expression of cyclin E1 (CCNE1) was associated with palbociclib resistance (interaction $P$ value $=0.002$ ) [10]. In addition, a research-based PAM50 intrinsic subtype predictor was also explored [3]. Similar to prior PAM50 studies in the advanced setting [12-14], the PALOMA-3 results confirmed that all the subtypes (i.e., Luminal A, Luminal B, HER2-enriched [HER2-E], and Basal-like), as well as the normal-like group, are identified within HR+/HER2negative disease. However, the PALOMA-3 PAM50 analysis did not confirm the prognostic value of these entities. This is in contrast with PAM50 retrospective data of 905 patients from two Phase III clinical trials in AI-naïve (EGF3008 study) [12] and AI-resistant (BOLERO-2) [13] HR+/HER2negative advanced breast cancer, where the non-Luminal subtypes, as a group, were strongly associated with poor progression-free survival and overall survival, regardless of 
Fig. 1 CCNE1 mRNA expression by subtype across four HR+/HER2-negative breast cancer data sets. a EGF3008 data set $(n=644)$ [12]; b BOLERO-2 data set $(n=261)$ [13]; c METABRIC data set $(n=1227)$ [15]; d MDACC508 data set $(n=306)[16,17]$
A

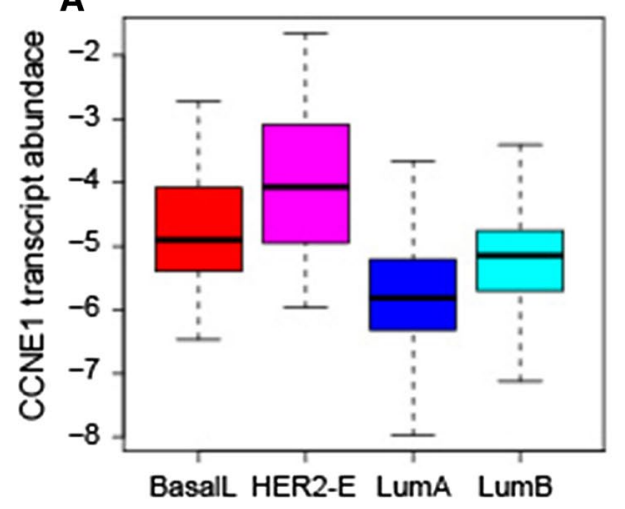

C

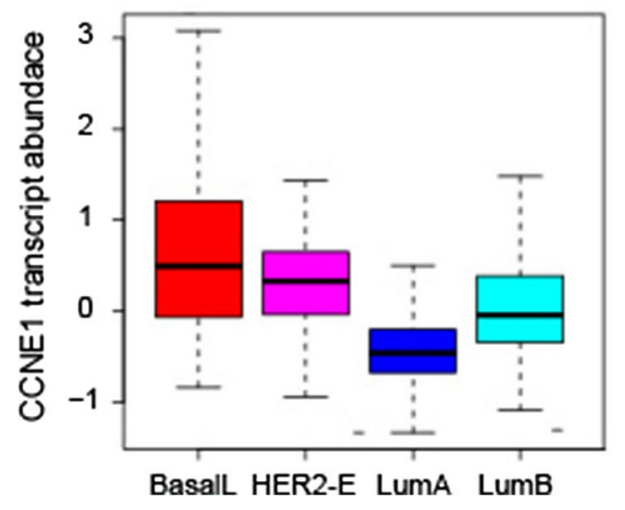

B

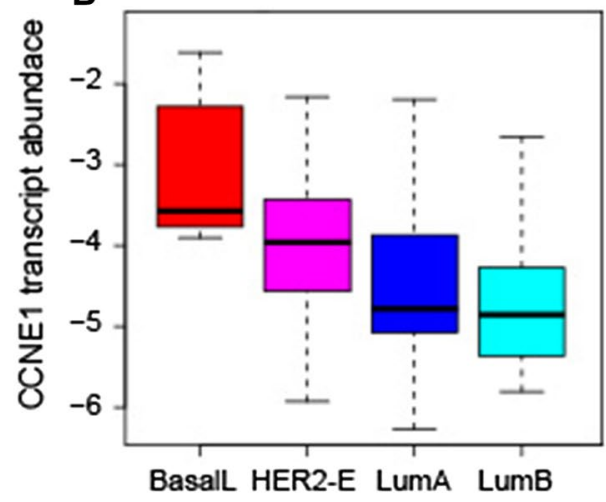

D

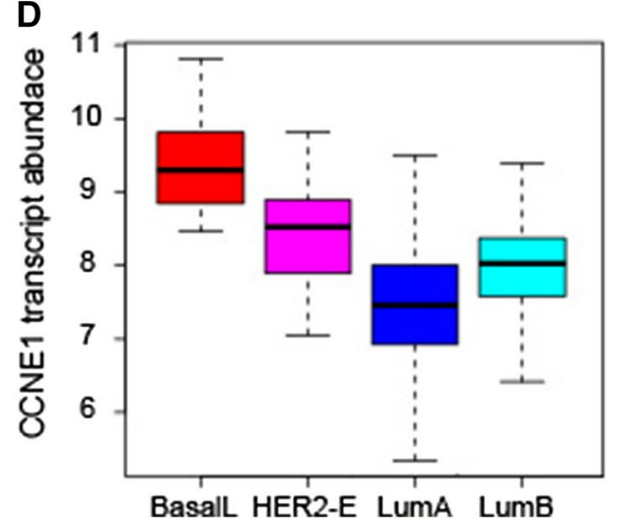

treatment and other clinical-pathological variables. In PALOMA-3 [10], the non-Luminal subtypes had a similar PFS as Luminal B disease with and without palbociclib (e.g., 5.5 versus 3.5 months in the fulvestrant-only arm and 9.5 versus 9.2 months in the combination arm).

In our opinion, the lack of prognostic value of intrinsic subtyping in PALOMA-3 compared to other studies needs attention, and may simply be due to technical (not biological) features. One important aspect to highlight is that the research-based subtyping analytical method used by Turner et al. [10], called Absolute Assignment of Breast Cancer Intrinsic Molecular Subtype (AIMS) [8], suffers from inherent analytical limitations [6] that arise from the use of relative expression measures in the absence of controls; in addition, Turner et al. [10] used RNAseq, and not nCounter, thus highlighting a second significant methodological difference. All of these limitations are well acknowledged by the authors as they attempt to identify the most appropriate subtype call for each sample. However, the plot of CCNE1 mRNA expression by intrinsic molecular subtype (Fig. 5c in Turner et al. [10]) suggests that the subtype calls may be biased. This plot shows that the expression of CCNE1 is highest in Luminal B than HER2-E subtypes in PALOMA-3. This observation is not seen across four independent studies in HR+/HER2negative disease (Fig. 1), including BOLERO-2, where the expression of CCNE1 is higher in HER2-E disease compared to Luminal B disease. Importantly, the AIMS approach has $\sim 50 \%$ concordance between Luminal B and HER2-E subtypes, and that the misclassifications are typically between these two subtypes [8]. Thus, a future re-analysis of the PAM50 test in the PALOMA-3 trial using the standardized PAM50/Prosigna assay seems well justified.

To conclude, research-based PAM50 subtyping needs particular attention and, if no controls are used to ensure proper subtype calling, the results should either not be reported, since they might be misleading or should be reported by proper acknowledgement of all the limitations and by clearly stating that this a research-based version of the PAM50 assay. This is the same that we would ask if one used a drug "similar" to paclitaxel, such as docetaxel, to draw conclusions regarding paclitaxel, or if one claims to have used a biosimilar drug in their study without showing any type of analytical similarity to the originator.

\section{Compliance with ethical standards}

Conflict of interest AP reports consulting and lecture fees from Nanostring Technologies, Inc. JP disclosed that he is an inventor of the 
pending patents on which the Prosigna Assay is based and a paid consultant of NanoString Technologies, Inc.

\section{References}

1. Perou CM, Sorlie T, Eisen MB, et al. Molecular portraits of human breast tumours. Nature. 2000;406:747-52.

2. Bastien RRL, Rodríguez-Lescure Á, Ebbert MTW, et al. PAM50 breast cancer subtyping by RT-qPCR and concordance with standard clinical molecular markers. BMC Med Genomics. 2012;5:44.

3. Parker JS, Mullins M, Cheang MCU, et al. Supervised risk predictor of breast cancer based on intrinsic subtypes. J Clin Oncol. 2009;27:1160-7.

4. Sørlie T, Perou CM, Tibshirani R, et al. Gene expression patterns of breast carcinomas distinguish tumor subclasses with clinical implications. Proc Natl Acad Sci. 2001;98:10869-74.

5. Hu Z, Fan C, Oh DS, et al. The molecular portraits of breast tumors are conserved across microarray platforms. BMC Genomics. 2006;7:96.

6. Perou CM, Parker JS, Prat A, et al. Clinical implementation of the intrinsic subtypes of breast cancer. Lancet Oncol. 2010;11:718-9.

7. Pogue-Geile KL, Song N, Jeong J-H, et al. Intrinsic subtypes, PIK3CA mutation, and the degree of benefit from adjuvant trastuzumab in the NSABP B-31 trial. J Clin Oncol. 2015;33:1340-7.

8. Paquet ER, Hallett MT. Absolute assignment of breast cancer intrinsic molecular subtype. J Nat Cancer Inst. 2014;107:2014.

9. Gendoo DMA, Ratanasirigulchai N, Schröder MS, et al. Genefu: an R/Bioconductor package for computation of gene expression-based signatures in breast cancer. Bioinformatics. 2015;32:1097-9.

10. Turner NC, Liu Y, Zhu Z, et al. Cyclin E1 expression and palbociclib efficacy in previously treated hormone receptor-positive metastatic breast cancer. J Clin Oncol. 2019;37:1169.
11. Turner NC, Slamon DJ, Ro J, et al. Overall survival with palbociclib and fulvestrant in advanced breast cancer. N Engl J Med. 2018;379:1926-36.

12. Prat A, Cheang MCU, Galván P, et al. Prognostic value of intrinsic subtypes in hormone receptor-positive metastatic breast cancer treated with letrozole with or without lapatiniblapatinib therapy and subtype in hormone receptor-positive metastatic breast cancerlapatinib therapy and subtype in hormone receptor-positive metastatic breast cancer. JAMA Oncol. 2016;2:1287-94.

13. Prat A, Brase JC, Cheng Y, et al. Everolimus plus exemestane for hormone receptor-positive advanced breast cancer: a PAM50 intrinsic subtype analysis of BOLERO-2. Oncologist. 2019;24(7):893-900. https://doi.org/10.1634/theoncolog ist.2018-0407.

14. Cejalvo JM, Martínez de Dueñas E, Galvan P, et al. Intrinsic subtypes and gene expression profiles in primary and metastatic breast cancer. Cancer Res. 2017;77:2213-21.

15. Curtis C, Shah SP, Chin S-F, et al. The genomic and transcriptomic architecture of 2000 breast tumours reveals novel subgroups. Nature. 2012;486:346.

16. Prat A, Fan C, Fernández A, et al. Response and survival of breast cancer intrinsic subtypes following multi-agent neoadjuvant chemotherapy. BMC Med. 2015;13:303-303.

17. Hatzis C, Pusztai L, Valero V, et al. A genomic predictor of response and survival following taxane-anthracycline chemotherapy for invasive breast cancer. JAMA. 2011;305:1873-81.

Publisher's Note Springer Nature remains neutral with regard to jurisdictional claims in published maps and institutional affiliations. 